\title{
Numerical Solution of a Certain Class of Singular Fredholm Integral Equations of the First Kind via the Vandermonde Matrix
}

\author{
E. S. Shoukralla * and M. A. Markos** \\ * Prof. of Eng. Math., Faculty of Electronic Engineering, Menoufia University, Egypt. \\ ** Assistant Lecturer, Faculty of Electronic Engineering, Menoufia University, Egypt.
}

\begin{abstract}
In this paper, a computational method is presented to solve potential-type Fredholm integral equations of the first kind, equations in which the unknown functions are singular at the endpoints of the integration domain, in addition to the weakly singular logarithmic kernels. This method provides a numerical solution based on the Newton interpolation technique via the Vandermonde matrix, which can accommodate an approximation of the unknown function, in such a manner that its singularity is easily removed, as well as the removal of kernel singularity. In addition, the Gauss-Legendre formula is adapted and applied for the computations of the obtained convergent integrals. Thus, the obtained numerical solution is equivalent to the solution of an algebraic equation in matrix form without applying the collocation method. The numerical solutions of the illustrated example are strongly converging to the exact solution for all values of $x \leq 1$ including the end-points \pm 1 whereas the exact solution fails to find the functional values at these end-points; which ensures the powerful and high accuracy of the presented computational technique.
\end{abstract}

Keywords-Electro-optics, electromagnetism, Fredholm integral equations, well-posed, singular, logarithmic kernel.

\section{INTRODUCTION}

The solutions to the boundary value problems associated for example, with Laplace equations, Helmholtz equations, and others, which are subject to Dirichlet or Neumann conditions, often lead to the solution of an equivalent Fredholm boundary integral equation [1]-[3]. Fredholm integral equations of the first kind with singular logarithmic or Cauchy kernels arise in many fields such as electron-optics, potential theory, and electromagnetism in the case of the replacement of a harmonic function by single-layer potentials. In many cases, especially in open boundaries cases, a singular integral equivalent equation is obtained. The singularities are due to the singularity of the kernel, the singularity of the unknown function or both singularities [4]-[6].

Many methods are published for the numerical and approximate solutions to Fredholm integral equations of the second kind which can be applied to solve the first kind equation [7]-[8]. There are other methods to solve Fredholm integral equations of the first kind with weakly logarithmic or Cauchy type singular integral equations [9]-[11]. The reformulating of the Dirichlet boundary value problem for the Laplace equation of mass distribution on an open contour in the plane give rise to an equivalent potential-type Fredholm integral equation of the first kind; with singular unknown function at the endpoints of the integration domain, in addition to the weakly singular logarithmic kernel. Methods for solving this class of equations have been published in [12]-[15], where the authors used orthogonal functions, the economized monic Chebyshev polynomials and monic Chebyshev polynomials. Seifi et al [10] presented an effective numerical method to solve Cauchy type singular Fredholm integral equations (CSFIEs) of the first kind by using the collocation technique based on Bernstein polynomials for the approximation of the solution of various cases of CSFIEs. Eshkuvatov et al [11] proposed a study of efficient approximate methods for solving Cauchy type singular integral equations (CSIEs) of the first kind, over a finite interval by using Chebyshev polynomials of the first, second, third, and fourth kinds. Shoukralla [12], the author presented a method based on the treatment of the singularity of the unknown function by changing the variables of the singular part and approximated the regular part by Chebyshev polynomials. The kernel was approximated twice by Chebyshev polynomials and by the change of variable. This complicated the procedure strikingly and failed to obtain the solution at the end-points of the open arc. Shoukralla et al [13] presented a method based on the economized Chebyshev polynomials with analytical treatments of the singularity. The method failed to obtain the solution at the end-points of the open arc. Additionally, Shoukralla et al [14] solved the same problem by applying the monic Chebyshev polynomials and collocation method, but the solution failed to give functional values at the endpoints of the integration domain. Later, Shoukralla et al [15] solved the same problem using Newton 
interpolation and collocation method, but the solution failed to give functional values at the endpoints of the integration domain as well as the exact solution.

This paper is devoted to establishing a computational method for solving the same equation without employing the collocation method and is capable to obtain the solution values at the endpoints of the integration domain, in addition to isolating the singularities of both the kernel and the unknown function. The proposed method improves the traditional Newton interpolation via the Vandermond matrix in such a manner that the monomial basis functions are easily separated from all the interpolation functions and the kernel. This technique proficient not only in producing the interpolation unknown function in a simple matrix form but also in removing all the singularities of the integral equation. The implementation of this goal is accomplished by utilizing a matrix-based algebra technique that facilitates many abbreviations so that the solution steps are significantly reduced, and the round off error is minimized. The unknown function is factorized into two functions, the first is a badlybehaved function and the second is a regular unknown function. The regular unknown function and the given data function are then interpolated by Newton interpolating polynomials of the same degree in matrix forms, while the badly-behaved function is expanded into Maclaurin polynomial of the same degree. Furthermore, the weak singularity of the kernel is removed upon expanding its two parametric equations into their corresponding Taylor polynomials of the first degree about the singular parameter. The resulted integrals are computed using the given adapted Gauss-Legendre quadrature formula. Consequently, we have concluded that the required solution is equivalent to solve an algebraic equation. Solving this algebraic equation by the comparison of the coefficients gives the coefficients of the unknown function and thus the unknown function itself can be found. The obtained numerical solutions of the illustrated example are strongly converging to the exact solution and gives values to the solution at the endpoints of the integration domain.

\section{NEWTON INTERPOLATION METHOD VIA VANDERMONDE MATRIX}

The density function of the solution of the Dirichlet openboundary value problem for two-dimensional Laplace equation of an electrostatic charged thin bar, which is symmetric about the $y$ - axis is equal the solution of a certain potential-type Fredholm integral equation of the first kind with weakly singular logarithmic kernel and whose unknown function is singular at the endpoints of the integration domain [13]. A certain class of such equations can be defined as

$$
\int_{\Gamma} \phi(x) k(x, y) d s_{x}=f(y) ; y \in \Gamma
$$

Here $\Gamma \in \square^{2}$ is a simple open contour that satisfies the Hölder condition $H^{\alpha}(\Gamma) ; 0<\alpha \leq 1, k(x, y)$ is the kernel such that $k(x, y)=\ln (1 /|d(x, y)|)$; where $d(x, y)$ denotes the distance between the two points $x, y$ on $\Gamma, \phi(x)$ is the undetermined singular unknown function defined on $\Gamma$, and $f(y) \in C(\Gamma)$ is the known given potential function. However, $\Gamma$ is parameterized as

$$
\left.\begin{array}{l}
x: x(\zeta), y(\zeta) ; a \leq \zeta \leq b ; \\
x^{\prime}(\zeta) \neq 0, y^{\prime}(\zeta) \neq 0 ; a<\zeta<b, \\
y: x(\tau), y(\tau) ; a \leq \tau \leq b ; \\
x^{\prime}(\tau) \neq 0, y^{\prime}(\tau) \neq 0 ; a<\tau<b,
\end{array}\right\}
$$

Therefore, integral Eq. (1) is transformed to

$$
\int_{a}^{b} \phi(\zeta) J(\zeta) k(\zeta, \tau) d \zeta=f(\tau) ; a \leq \tau \leq b
$$

Where $\xi, \tau$ are the parameters of the two points $x, y$ on $\Gamma, J(\xi)$ is the Jacobian of the parameterized open contour $\Gamma$, such that $J(\zeta)=\sqrt{\left(x^{\prime}(\zeta)\right)^{2}+\left(y^{\prime}(\zeta)\right)^{2}}$. For simplicity, let the integration domain be transformed to the normalized domain $[-1,1]$ by using the linear transform $\zeta=a+\frac{b-a}{2}(\xi+1)$. Hence Eq. (3), is transformed into

$$
\int_{-1}^{1} \phi(\xi) J(\xi) k(\xi, \tau) d \xi=f(\tau) ;-1 \leq \tau \leq 1
$$

Let us first redefine the given function $f(\tau)$ as a data function defined by $f\left(\tau_{i}\right)=f_{i}$ for $\tau_{i}=-1+i h$, for some step-size $h=\frac{2}{n}>0$ where $i=\overline{0, n}$. By $\tilde{f}(\tau)$ the Newton interpolation polynomial of degree $n$ that interpolates $f(\tau)$ at the nodes $\left\{\tau_{i}\right\}_{i=0}^{n-1}$ such that $\tilde{f}\left(\tau_{i}\right)=f_{i}$. In a matrix form, the Newton interpolation polynomial $N_{n}(\tau)$ takes the form

$$
[\tilde{f}(\tau)]=\mathrm{T}(\tau) \mathrm{A}
$$

where

$$
\begin{aligned}
& \mathrm{T}(\tau)= \\
& {\left[1 \quad\left(\tau-\tau_{0}\right) \cdots\left(\tau-\tau_{0}\right)\left(\tau-\tau_{1}\right) . .\left(\tau-\tau_{n-1}\right)\right]}
\end{aligned}
$$


and the column matrix $\mathrm{A}$ is defined by

$$
\left.\begin{array}{l}
\mathrm{A}=\left[a_{i}\right]_{i=0}^{n} ; a_{i}=\frac{\Delta^{i} f_{0}}{(i !)(h)^{i}} ; \\
\Delta^{i} f_{0}=\sum_{s=0}^{i}\left(\begin{array}{l}
i \\
s
\end{array}\right)(-1)^{i-s} f_{s} ; \Delta^{0} f_{0}=f_{0}
\end{array}\right\}
$$

Furthermore, the interpolation polynomial of $\tilde{f}(\tau)$ can then be rewritten via the Vandermonde matrix $\tilde{\mathrm{T}}$ in the following form

$$
\tilde{f}(\tau)=\Phi(\tau) \tilde{\mathrm{T}}^{-1} \mathrm{~F}
$$

where $\mathrm{F}$ is the known functional values column matrix $\mathrm{F}=\left[f_{i}\right]_{i=0}^{n}$, while the row matrix $\Phi(\tau)$ and the Vandermonde square matrix $\tilde{\mathrm{T}}$ are defined by

$$
\Phi(\tau)=\left[\tau^{i}\right]_{i=0}^{n}, \tilde{\mathrm{T}}=\left[\tau_{i j}\right]_{i, j=0}^{n} ; \tau_{i j}=\tau_{i}^{j}, \tau_{00}=1
$$

Now, the unknown function $\phi(\xi)$ is replaced by the product

$$
\phi(\xi)=v(\xi) u(\xi)
$$

where $u(\xi)$ is a regular unknown function, and $v(\xi)=\frac{1}{\sqrt{1-\xi^{2}}}$ expresses the singular behavior of the unknown function $\phi(\xi)$ near and at the end-points of the integration domain when $x \rightarrow \pm 1$. Suppose that $u(\xi)$ is given as a tabulated function $u\left(\xi_{i}\right)=u_{i}$ for the set of $n$ knots $\left\{\xi_{i}, u\left(\xi_{i}\right)\right\}_{i=0}^{n-1}$. Then, similar to the interpolation of $f(\tau), u(\xi)$ this may be replaced by its interpolation polynomial $\tilde{u}(\xi)$ in the matrix form

$$
[\tilde{u}(\xi)]=\Phi(\xi) \tilde{\mathrm{T}}^{-1} \mathrm{U}
$$

where $\mathrm{U}=\left[u_{i}\right]_{i=0}^{n}$ is the unknown coefficient column matrix to be determined. Now, the singular function $v(\xi)$ is expanded in Maclurin polynomial of the same degree $n$ in the matrix form

$$
[v(\xi)]=\mathrm{V} \Phi^{T}(\xi)
$$

where the row coefficient matrix $\mathrm{V}$ is defined by

$$
\mathrm{V}=\left[v_{i}\right]_{i=0}^{n} ; v_{i}=\frac{v^{(i)}(0)}{i !} ; i=\overline{0, n}
$$

Substituting Eqs. (11), (12) into Eq. (10) gives

$$
\phi(\xi)=\mathrm{V} \tilde{\Phi}(\xi) \tilde{\mathrm{T}}^{-1} \mathrm{U}
$$

where the square matrix $\tilde{\Phi}$ is given by

$$
\begin{aligned}
& \tilde{\Phi}(\xi)= \\
& \Phi^{T}(\xi) \Phi(\xi)=\left[\varphi_{i j}(\xi)\right]_{i, j=0}^{n} ; \varphi_{i j}=\xi^{i+j}
\end{aligned}
$$

Moreover, the logarithmic singular kernel $k(x, y)=\ln (1 /|d(x, y)|)$, where $d(x, y)$ is the distance from the parametric point $x(x(\xi), y(\xi))$ to the parametric point $y(x(\tau), y(\tau))$, can be rewritten in the form

$$
\left.\begin{array}{l}
k(\xi, \tau)= \\
\ln \left(1 / \sqrt{(x(\xi)-x(\tau))^{2}+(y(\xi)-y(\tau))^{2}}\right) ; \\
-1 \leq \xi, \tau \leq 1
\end{array}\right\}
$$

From Eq. (16) it turns out that the singularities of $k(x, y)$ occurs when $\xi \rightarrow \tau$. To isolate this singular behavior, both $x(\xi), y(\xi)$ are approximated using Taylor polynomial of the first degree about the singular parameter $\tau$. Thus, we have

$$
\left.\begin{array}{l}
x(\xi)=x(\tau)+(\xi-\tau) x^{\prime}(\tau) \\
y(\xi)=y(\tau)+(\xi-\tau) y^{\prime}(\tau)
\end{array}\right\}
$$

By substituting Eq. (17) into Eq. (16), we obtain $k(\xi, \tau)=\ln (1 / \alpha|\xi-\tau|) ;-1 \leq \xi, \tau \leq 1$,

$\alpha=\sqrt{\left(x^{\prime}(\tau)\right)^{2}+\left(y^{\prime}(\tau)\right)^{2}}$

Finally, substituting Eqs. (8), (14), and (18) into Eq. (4), gives

$$
\mathrm{V} \Psi(\tau) \tilde{\mathrm{T}}^{-1} \mathrm{U}=\Phi(\tau) \tilde{\mathrm{T}}^{-1} \mathrm{~F} ;-1 \leq \tau \leq 1
$$

Here

$$
\left.\begin{array}{l}
\Psi(\tau)=\left[\psi_{i j}(\tau)\right]_{i, j=0}^{n} ; \\
\psi_{i j}(\tau)= \\
\int_{-1}^{1} J(\xi) \xi^{i+j} \ln (1 / \alpha|\xi-\tau|) d \xi ; i, j=\overline{0, \mathrm{n}}
\end{array}\right\}
$$

By applying the apdative $m$ - nodes Gauss-Legendre formula

$$
\int_{a}^{b} f(x) d x=\sum_{s=1}^{m} \delta_{s} f\left(\omega_{s}\right)
$$

where the weights $\delta_{s}$, and the nodes $\omega_{s}$ are given by

$$
\left.\begin{array}{l}
\delta_{s}=\frac{b-a}{\left(1-\alpha_{s}^{2}\right)\left(P_{m}^{\prime}\left(\alpha_{s}\right)\right)^{2}}, \\
\omega_{s}=\frac{b-a}{2} \alpha_{s}+\frac{b+a}{2} \forall s=\overline{1, m} ; \sum_{s=1}^{m} \delta_{s}=2
\end{array}\right\}
$$


and $\alpha_{s}$ are the roots of Legendre polynomial of degree $m$ defined on $[-1,1]$, the integrals $\psi_{i j}(\tau)$ can be easily computed since they became proper integrals. By solving the matrix equation (19) using the undetermined coefficients method, the unknown matrix $\mathrm{U}$ can be found, and thereby, the unknown function $\phi(\xi)$ of Fredholm integral equation (4) can be obtained.

\section{COMPUTATIONAL RESULTS}

Example (1): The unknown density function $f(x)$ of a charged wire of length 2 , which is symmetric about $y$-axis in the plane with constant data function equal to 1 , can be found by solving the weakly singular Fredholm integral equation of the first kind.

$$
\int_{-1}^{1} f(x) \ln \frac{1}{|x-y|} d x=1 ;-1 \leq y \leq 1
$$

whose exact solution [13] is given by

$$
f(x)=\frac{1}{\pi \ln (2) \sqrt{1-x^{2}}} ;-1<x<1
$$

Using the presented method, the numerical solutions of Eq. (23) are obtained. The computations were made using MATLAB and the CPU time was equal to 1.59 second for $n=4$. Moreover, the solution at the end-points $x= \pm 1$ had been computed to be as follows: $f( \pm 1)=1.2969,1.2742$ and 1.2491 for $n=2,3$, and 4 respectively. In Table I, a comparison is made between the exact solution denoted by $E\left(x_{i}\right)$ and the obtained numerical solutions $\phi_{n}\left(x_{i}\right)$ for $n=2,3$ and 4 respectively, where $n$ denotes the degree of the Newton interpolation polynomial. The number of GaussLegendre's nodes are chosen $m=20$. In table II the absolute error estimations $R_{n}\left(x_{i}\right)$ for $n=2,3$ and 4 respectively are given. In figures 1,2 , and 3 plotted are the graph of the exact solution $E\left(x_{i}\right)$ with the graphs of the obtained numerical solutions $\phi_{n}\left(x_{i}\right)$ for $n=2,3$ and 4 respectively.

Table 1. A comparison of the numerical solutions $\phi_{n}\left(x_{i}\right)$ with the exact solution $E\left(x_{i}\right)$.

\begin{tabular}{|c|c|c|c|c|c|}
\hline$i$ & $x_{i}$ & $E\left(x_{i}\right)$ & $\phi_{2}\left(x_{i}\right)$ & $\phi_{3}\left(x_{i}\right)$ & $\phi_{4}\left(x_{i}\right)$ \\
\hline 0 & -0.9000 & 1.0535 & 1.0557 & 1.0543 & 1.0438 \\
1 & -0.8000 & 0.7654 & 0.7670 & 0.7660 & 0.7583 \\
2 & -0.7000 & 0.6430 & 0.6444 & 0.6435 & 0.6371 \\
3 & -0.6000 & 0.5740 & 0.5752 & 0.5745 & 0.5687 \\
4 & -0.5000 & 0.5303 & 0.5314 & 0.5307 & 0.5254 \\
5 & -0.4000 & 0.5011 & 0.5021 & 0.5014 & 0.4964 \\
\hline
\end{tabular}

\begin{tabular}{|c|c|c|c|c|c|}
\hline 6 & -0.3000 & 0.4814 & 0.4824 & 0.4818 & 0.4769 \\
7 & -0.2000 & 0.4687 & 0.4697 & 0.4691 & 0.4644 \\
8 & -0.1000 & 0.4615 & 0.4625 & 0.4619 & 0.4573 \\
9 & 0.0000 & 0.4592 & 0.4602 & 0.4596 & 0.4550 \\
10 & 0.1000 & 0.4615 & 0.4625 & 0.4619 & 0.4573 \\
11 & 0.2000 & 0.4687 & 0.4697 & 0.4691 & 0.4644 \\
12 & 0.3000 & 0.4814 & 0.4824 & 0.4818 & 0.4769 \\
13 & 0.4000 & 0.5011 & 0.5021 & 0.5014 & 0.4964 \\
14 & 0.5000 & 0.5303 & 0.5314 & 0.5307 & 0.5254 \\
15 & 0.6000 & 0.5740 & 0.5752 & 0.5745 & 0.5687 \\
16 & 0.7000 & 0.6430 & 0.6444 & 0.6435 & 0.6371 \\
17 & 0.8000 & 0.7654 & 0.7670 & 0.7660 & 0.7583 \\
18 & 0.9000 & 1.0535 & 1.0557 & 1.0543 & 1.0438 \\
\hline
\end{tabular}

Table 2. The absolute error estimations $R_{n}\left(x_{i}\right)$.

\begin{tabular}{|c|c|c|c|c|}
\hline$i$ & $x_{i}$ & $R_{2}\left(x_{i}\right)$ & $R_{3}\left(x_{i}\right)$ & $R_{4}\left(x_{i}\right)$ \\
\hline 0 & 0.0000 & $9.5569 \mathrm{e}-4$ & $3.4983 \mathrm{e}-4$ & $4.2461 \mathrm{e}-3$ \\
1 & 0.1000 & $9.6051 \mathrm{e}-4$ & $3.5154 \mathrm{e}-4$ & $4.2675 \mathrm{e}-3$ \\
2 & 0.2000 & $9.7540 \mathrm{e}-4$ & $3.5699 \mathrm{e}-4$ & $4.3336 \mathrm{e}-3$ \\
3 & 0.3000 & $1.0018 \mathrm{e}-3$ & $3.6667 \mathrm{e}-4$ & $4.4511 \mathrm{e}-3$ \\
4 & 0.4000 & $1.0427 \mathrm{e}-3$ & $3.8164 \mathrm{e}-4$ & $4.6328 \mathrm{e}-3$ \\
5 & 0.5000 & $1.1035 \mathrm{e}-3$ & $4.0389 \mathrm{e}-4$ & $4.9029 \mathrm{e}-3$ \\
6 & 0.6000 & $1.1946 \mathrm{e}-3$ & $4.3722 \mathrm{e}-4$ & $5.3076 \mathrm{e}-3$ \\
7 & 0.7000 & $1.3382 \mathrm{e}-3$ & $4.8989 \mathrm{e}-4$ & $5.9457 \mathrm{e}-3$ \\
8 & 0.8000 & $1.5928 \mathrm{e}-3$ & $5.8307 \mathrm{e}-4$ & $7.0768 \mathrm{e}-3$ \\
9 & 0.9000 & $2.1925 \mathrm{e}-3$ & $8.0255 \mathrm{e}-4$ & $9.7412 \mathrm{e}-3$ \\
\hline
\end{tabular}

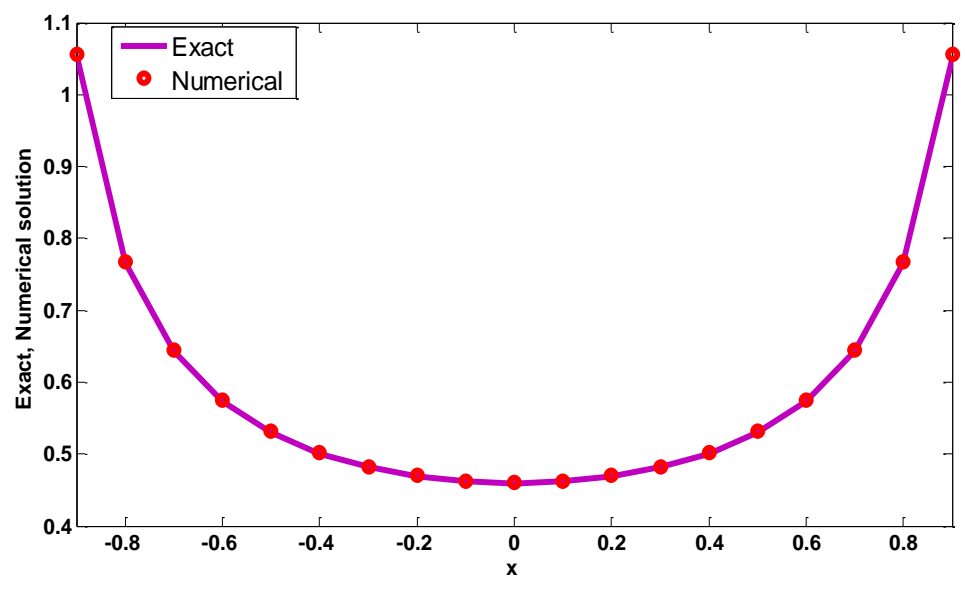

Fig. 1. The graphs of $\phi_{2}\left(x_{i}\right)$ and $E\left(x_{i}\right)$. 


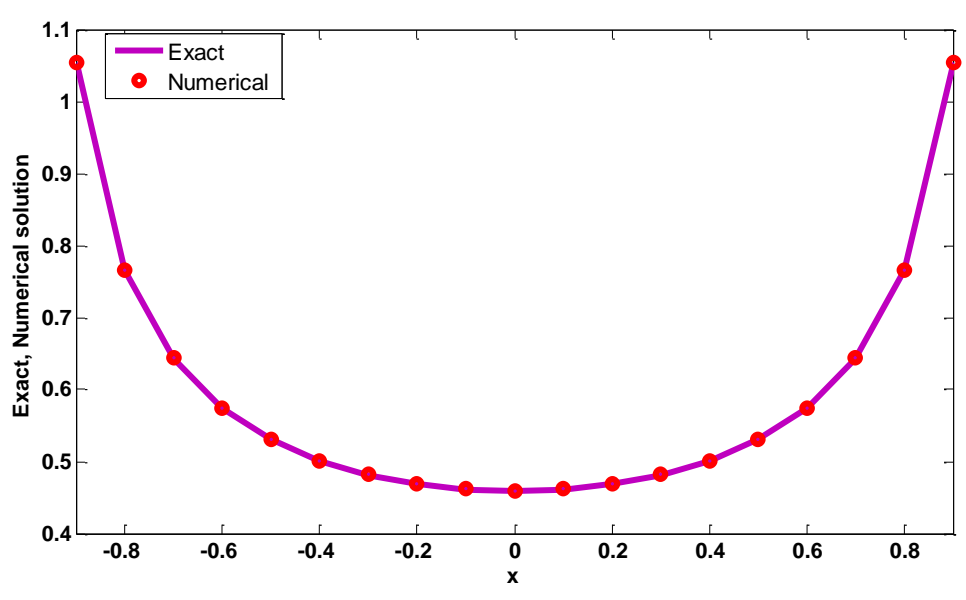

Fig. 2. The graphs of $\phi_{3}\left(x_{i}\right)$ and $E\left(x_{i}\right)$.

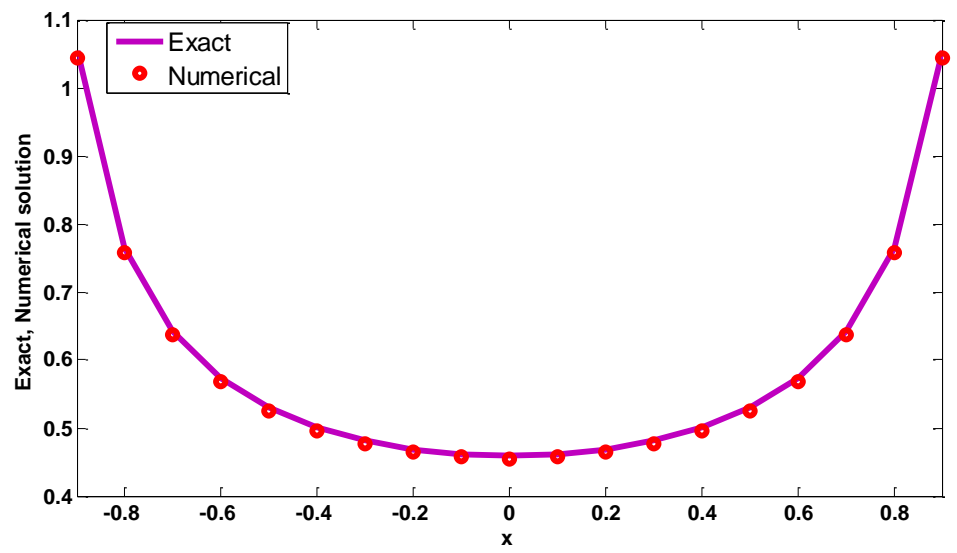

Fig. 3. The graphs of $\phi_{4}\left(x_{i}\right)$ and $E\left(x_{i}\right)$.

Fig. 1, Fig. 2, and Fig. 3 give a comparison between the exact solutions denoted by $\tilde{E}_{i}\left(x_{i}\right)$ with the numerical solutions for $n=2,3$ and 4 respectively, which ensures a superior accuracy of the given numerical solutions.

Example (2): Consider the weakly singular Fredholm integral equation of the first kind with singular logarithmic kernel.

$$
\int_{0}^{2 \pi} \Phi(t) \ln \left(2 a\left(\sin \frac{|t-t *|}{2}\right)\right) d t=-\frac{\pi}{2} \cos (2 t *)
$$

Here, the arc $\Gamma$, defined in Eq. (1) is a circle with radius $a, x=a \cos (t), y=a \sin (t), 0 \leq t \leq 2 \pi$. The exact solution is $\Phi(t)=\cos (2 t)$ [16]. In general, this solution is unique, while for $a=1$ (a unit circle) the solution is $\Phi(t)=\cos (2 t)+a_{0}$, where $a_{0}$ is an arbitrary constant. In table III, the obtained numerical solutions by using Newton interpolation polynomial for $n=18$ and 20 respectively are compared with the exact solution denoted by $\tilde{E}_{i}\left(x_{i}\right)$. In Figure 4, plotted the graph of the exact solution and Newton interpolation polynomial numerical solutions for $n=20$ and $m=20$.

Table 3. A comparison between the exact solution denoted by $\tilde{E}_{i}\left(x_{i}\right)$ with the Newton interpolation polynomial numerical solutions for $n=18$ and 20 respectively.

\begin{tabular}{|c|c|c|c|c|}
\hline$i$ & $x_{i}$ & $\tilde{E}_{i}\left(x_{i}\right)$ & $n=18$ & $n=20$ \\
\hline 0 & 0 & 1.0000 & 1.0000 & 1.0000 \\
1 & $\pi / 4$ & 0.0000 & 0.0000 & 0.0000 \\
2 & $\pi / 2$ & -1.0000 & -0.9998 & -0.9999 \\
3 & $3 \pi / 4$ & 0.0000 & -0.0002 & 0.0000 \\
4 & $\pi$ & 1.0000 & 0.9998 & 0.9999 \\
5 & $5 \pi / 4$ & 0.0000 & 0.0003 & 0.0000 \\
6 & $3 \pi / 2$ & -1.0000 & -0.9998 & -0.9999 \\
7 & $7 \pi / 4$ & 0.0000 & -0.0005 & 0.0000 \\
8 & $2 \pi$ & 1.0000 & 0.9998 & 0.9999 \\
& & & & \\
\hline
\end{tabular}

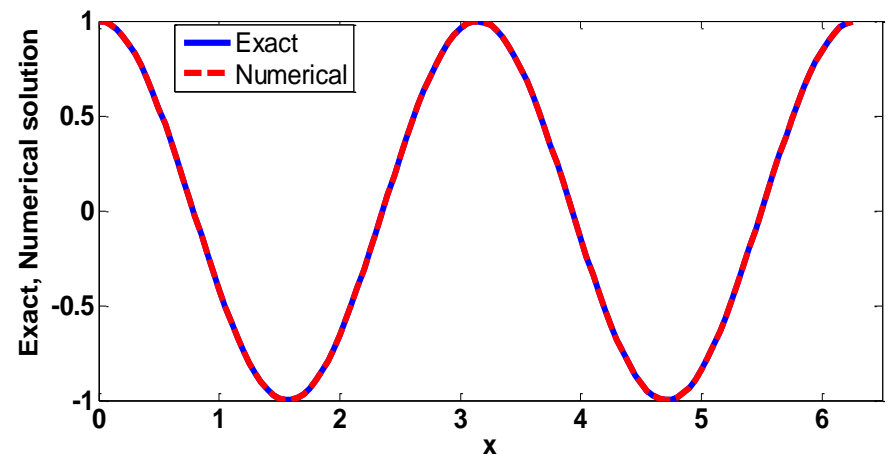

Fig. 4. A comparison between the exact solutions with the Newton interpolation polynomial numerical solution for $n=20$.

\section{CONCLUSION}

A computational method has been investigated for the numerical solution of a certain potential-type Fredholm integral equation of the first kind with a singular unknown function at the end-points of the integration domain and has a weakly singular logarithmic kernel. The presented method is based on Newton interpolation in a matrix form, in addition to the analytical treatment of the singularities of both the kernel and the unknown function without changing the variables. The singularity of the singular unknown function was treated by considering it as a product of two functions. The first was given in a closed form that expresses its singular behavior, while the second, which is a regular function, was interpolated using Newton interpolation in a matrix form. The singularity of the kernel was treated analytically by expanding the two parametric functions of the parameterized kernel via Taylor polynomial of the first degree about the singular parameter. Furthermore, an adaptive Gauss-Legendre formula was 
applied, and matrix algebra was utilized, in such a manner that the unknown function was found by solving a linear algebraic equation in a matrix form. The advantage of this method is an inevitable result of adapting a matrix-based algebra technique, where we were able to obtain a monomial basis matrix that facilitated the solution, reduced the steps of the solution and led to its superior accuracy compared with the other methods.

\section{REFERENCES}

[1] A. M. Simoes, "Fredholm Characterization for a Wave Diffraction Problem with Higher Order Boundary Conditions: Impedance Case", WSEAS Transactions on Mathematics, Vol. 13, pp. 535-546, 2014.

[2] R. Chapko and B. T. Johansson, "An Alternating PotentialBased Approach To The Cauchy Problem For The Laplace Equation In A Planar Domain With A Cut", Computational Methods in Applied Mathematics, Vol. 8, No. 4, pp. 315-335, 2008.

[3] E. S. Shoukralla, "Numerical solution of Helmholtz equation for an open boundary in space", Journal of appl. Math Modeling, Vol. 4, No. 21, pp. 231-232, 1997.

[4] Y. V. Shestopalov, E. V. Chernokozhin, "On the solution to integral equations with a logarithmic singularity of the kernel on several intervals of integration: elements of the spectral theory", Visnek, Kharkov National university, Ukraine. 1058 , 2013.

[5] E. Kendall, Atkinson, I. H. Sloan, "The numerical solution of first-kind logarithmic-kernel integral equations on smooth open arcs". Mathematics of Computation, Vol 56, pp.119-139, 1991.

[6] V. Domínguez, "High-order collocation and quadrature methods for some logarithmic kernel integral equations on open arcs", Journal of Computational \& Applied Mathematics. Vol. 16, pp.145-159, 2003.

[7] I. G. Burova, N. S. Domnin, A. E. Vezhlev, A. V. Lebedeva, A. N. Pakulina, "On the Solution of the Fredholm Equation of the Second Kind", WSEAS Transactions on Mathematics, Vol. 17, pp. 319-328, 2018.

[8] E. S. Shoukralla, H. Elgohary and B. M. Ahmed, "Barycentric Lagrange interpolation for solving Volterra integral equations of the second kind", Published in the 4th International conference on Advanced Technology and Applied Sciences (ICaTAS2019), Journal of Physics, England, Conference Series, 1447(2020),012002.

[9] Amit Setia, "Numerical solution of various cases of Cauchy type singular integral equation", Applied Mathematics and Computation, Vol. 230, pp. 200-207, 2014.

[10] A. Seifi, T. Lotfi, T. Allahviranloo and M. Paripour, "An effective collocation technique to solve the singular Fredholm integral equations with Cauchy kernel", Advances in Difference Equations, Vol. 280, pp.1-18, 2017.

[11] Z. K. Eshkuvatov, N. M. A. Nik Long, M. Abdulkawi, "Approximate solution of singular integral equations of the first kind with Cauchy kernel", Applied Mathematics Letters, Vol. 22, pp. 651-657, 2009.

[12] E. S. Shoukralla, "Approximate solution to weakly singular integral equations", Journal of appl. Math Modelling, Vol. 20, pp. 800-803, 1996.

[13] E. S. Shoukralla and M. A. Markos, "The economized monic Chebyshev polynomials for solving weakly singular Fredholm integral equations of the first kind" Asian-European Journal of Mathematics, vol. 12, no. 1, pp. 1-10, 2020.
[14] E. S. Shoukralla, M. Kamel, and M. A. Markos, "A new computational method for solving weakly singular Fredholm integral equations of the first kind" Published in the 13th IEEE International Conference on Computer Engineering and Systems (ICCES 2018). Cairo, Egypt on December, (IEEE Xplore), 2018.

[15] E. S. Shoukralla, W. A. El-Ganaini, and M. A. Markos, "Numerical solution of singular Fredholm integral equations of the first kind using Newton interpolation" Menoufia J. of Electronic Engineering Research (MJEER), vol. 28, no. 1, pp. $1-12,2019$.

[16] S. Christiansen, "Numerical solution of an integral equation with a logarithmic kernel”, BIT, Vol. 11, pp. 276-287, 1971.

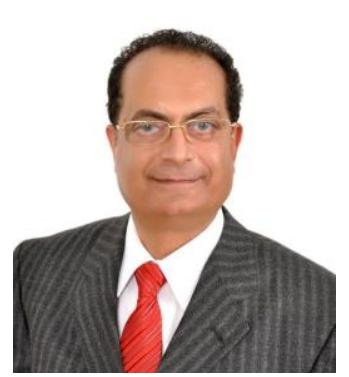

E. S. Shoukralla: Full Professor of Mathematics, currently works at the Dept. of Engineering Mathematics and Physics, Faculty of Electronic Engineering, Menoufia University, El-Menoufia, (Egypt). Formerly Head of the Dept., and the former Vice Dean of the Community Service and Environmental Development.

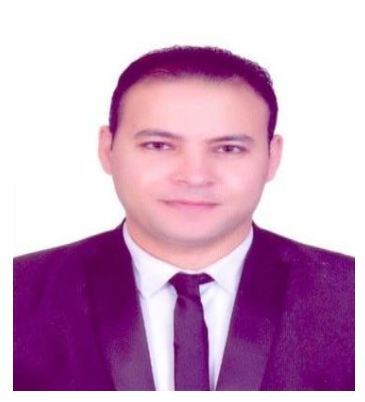

M. A. Markos: Assistant Lecturer of Engineering Mathematics at the Faculty of Electronic Engineering, Menoufia University, ElMenoufia, (Egypt). He earned his Bachelor in Industrial Electronics and Control Engineering (excellent with honor degree) on May 2013 from the Faculty of Electronic Engineering, Menoufia University (Egypt). He obtained his Master in Physics and Engineering Mathematics (excellent degree in pre-master courses) on February 2019 from Faculty of Electronic Engineering, Menoufia University (Egypt). His Thesis title is "Numerical solutions of singular Fredholm integral equations of the first kind". 